\title{
TÍCH HỢP THIẾT BỊ IMU VÀ GNSS THU NHẬN DŨ LIỆU SỬ DỤNG CÔNG NGHỆ TRAM THAM CHIẾU ẢO (VRS) TRÊN THIẾT BỊ BAY KHÔNG NGƯờI LÁI (UAV) PHỤC VỤ CÔNG TÁC THÀNH LẬP BẢN ĐỔ ĐỊA HİNH
}

\author{
LƯU HẢI ÂU, ĐẶNG XUÂN THỦY, NGÔ TH!̣ LIÊN \\ Viện Khoa học Đo đạc và Bản đồ
}

\section{Tóm tắt:}

Công tác thành lập bản đồ địa hình ưng dụng thiết bị bay không người lái (UAV) đã được úng dụng và đang dần trở lên phổ biến. Việc xác định chính xác các yếu tố định huớng ngoài của ảnh là một trong nhũng yếu tố quyết định đến độ chính xác của bản đồ địa hình cần thành lập thay vì thiết lập mạng luơoi khống chế ảnh. Bài báo này trình bày về vấn đề tích hợp thiết bị GNSS/IMU trên thiết bị bay không ngườ lái (UAV) kết hợp với công nghệ trạm tham chiếu ảo (VRS) để xác định các yếu tố định huớng ngoài của ảnh với độ chính xác đảm bảo thành lập bản đồ địa hình tỉ lệ lớn vớ số lượng điểm khống chế ảnh giảm tối đa.

\section{1. Đặt vấn đề}

Trên thế giới, công tác thành lập bản đồ địa hình ứng dụng thiết bị bay không người lái (UAV) đã rất phổ biến. Cùng với việc hoàn thiện thuật toán trong công tác xử lý nội nghiệp, các hệ thống phục vụ thu nhận dữ liệu ngoại nghiệp cũng phát triển mạnh mẽ. Quá trình phát triển hệ thống phục vụ thu nhận dữ liệu ngoại nghiệp trên thế giới hiện nay diễn ra theo các hướng như: tăng tính cơ động của hệ thống thiết bị, tăng số lượng và chất lượng dữ liệu thu nhận, giảm giá thành...Trong xu thế đó, các hãng sản xuất hệ thống thu nhận dữ liệu hàng đầu hiện nay như Trimble - Mỹ, Sensefly - Thuy Sỹ, Aeromao Canada... đã phát triển những hệ thống thiết bị được ứng dụng phổ biến trong công tác thu nhận dữ liệu phục vụ thành lập bản đồ địa hình các loại tỉ lệ.

Mặc dù mức độ tự động hóa của công nghệ sử dụng thiết bị bay không người lái trong thành lập bản đồ ngày càng được cải thiện và hiện nay nhiều phầm mềm thương mại đã đạt tới mức tự động hóa gần như hoàn toàn trong một số công đoạn thành lập bản đồ bằng phương pháp đo ảnh như: Định hướng tăng dày, thành lập mô hình số độ cao, thành lập ảnh trực giao nhưng hiện nay các tham số tham số định hướng ngoài của ảnh vẫn chưa được sử dụng rộng rãi và triệt để trong công tác thành lập bản đồ trên thế giới và ở nước ta. Chưa có thiết bị GNSS có thể tính tọa độ tâm ảnh chính xác. Hơn nữa các chương trình, phần mềm xử lý khi đến tay người dùng là hoàn toàn tự động và đóng kín. Do đó để nắm bắt và làm chủ được công nghệ cần thiết phải có các nghiên cứu về các thuật toán, chương trình, phần mềm áp dụng cho hệ thống UAV.

Nhằm tăng tính cơ động, tăng số lượng và chất lượng dữ liệu của hệ thống thu nhận dữ liệu, các hãng sản xuất trên thế giới đang phát triển theo hướng tích hợp các công nghệ mới trong hệ thống thu nhận dữ liệu như: tích hợp công nghệ định vị vệ tinh - phương pháp đo động, thiết bị định vị quán tính và công nghệ mới trong camera...Qua đó, dữ liệu thu được trong công tác ngoại nghiệp ngày càng gia tăng về số lượng, chủng loại và có độ tin cậy cao.

\section{Các kết quả nghiên cứu}

2.1. Giới thiệu hệ thống tích hợp GNSSIMU-UAV

(Xem hinh 1) 


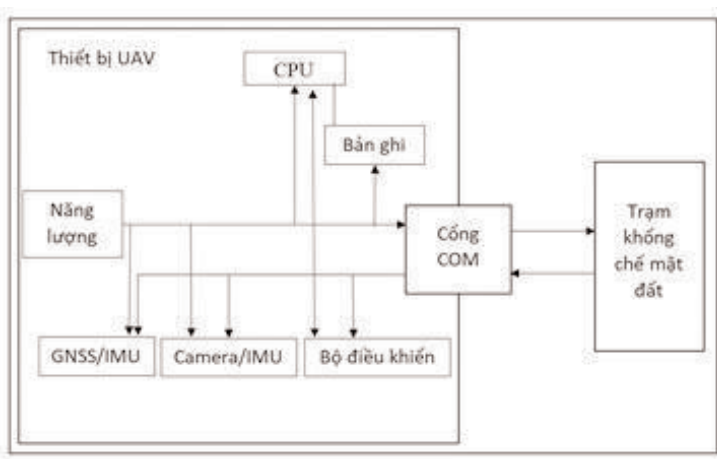

Hình 1: Mô hình tích hơp GNSS-IMU-UAV thống:

Thông số các thiết bị thành phần của hệ

a) IMU là loại cảm biến PMU 6050 với các thông số:

- Điện áp sử dụng: 3 5VDC

- Điện áp giao tiếp: 3 5VDC

- Chuẩn giao tiếp: $\mathrm{I} 2 \mathrm{C}$

- Giá trị Gyroscopes trong khoảng: +/- 250 50010002000 degree/sec

- Giá trị Acceleration trong khoảng: +/- 2g, +/- 4g, +/- 8g, +/- $16 \mathrm{~g}$

b) Thiết bị định vị GNSS: (Xem bảng 1) c) Thiết bị bay không người lái: (Xem bảng 2, hinh 2)
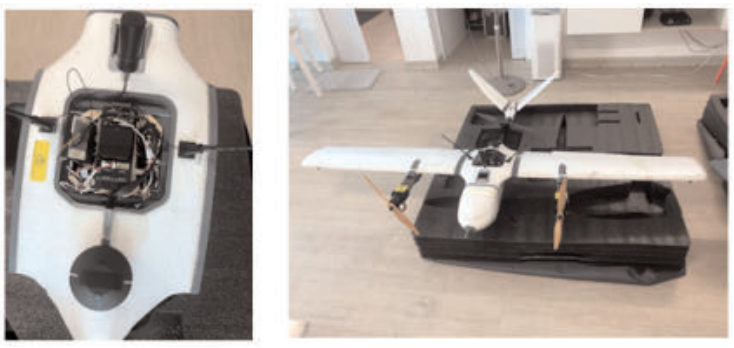

Hình 2: Hệ thống tích hợp GNSS-IMU trên UAV VTOL

\subsection{Thử nghiệm}

Bãi hiệu chuẩn phục vụ công tác xác định các thông số độ lệch chuẩn của hệ thống thiết bị được xây dựng tại khu vực thôn Cổ Rùa, xã Phú Mãn, huyện Quốc Oai, thành phố Hà Nội với diện tích 64 ha. Trong đó xây dựng 36 mốc khống chế mặt bằng và khống chế độ cao phủ trùm khu đo. Các mốc khống chế được đo đạc chi tiết theo hệ tọa độ quốc gia VN-2000 và hệ độ cao Nhà nước (Hòn Dấu). Các điểm khống chế mặt bằng này được tận dụng làm mốc khống chế độ cao, được đo nối với hệ độ cao Nhà nước (Hòn Dấu) theo tiêu chuẩn dẫn thủy chuẩn hạng IV.

Bảng 1: Thông số thiết bị GNSS

\begin{tabular}{|c|c|c|}
\hline STT & \multicolumn{2}{|c|}{ Thông số kỹ thuật } \\
\hline 1 & $\begin{array}{l}\text { Số lượng kênh thu đồng } \\
\text { thời }\end{array}$ & 192 kênh \\
\hline 2 & Hệ thống vệ tịnh định vị & $\begin{array}{l}\text { GPS L1+L2, } \\
\text { Beidou B1+B3 }\end{array}$ \\
\hline 3 & $\begin{array}{l}\text { Công nghệ cài chính } \\
\text { RTK }\end{array}$ & $\begin{array}{l}\text { Có hố trợ, đạt sai số đến } \\
\text { centimet }\end{array}$ \\
\hline 4 & Tần số xuất tọa độ & $\begin{array}{l}\text { Tối đa } 10 \mathrm{~Hz} \text { (10 vị trí trong } 1 \\
\text { giây) }\end{array}$ \\
\hline 5 & $\begin{array}{l}\text { Độ chính xác khi đo độc } \\
\text { lập }\end{array}$ & $\begin{array}{l}\text { Phương ngang: } 1,5 \mathrm{~m} \text {, phương } \\
\text { đứng } 3,0 \mathrm{~m}\end{array}$ \\
\hline 6 & Độ chính xác PPK & $\begin{array}{l}\text { P.ngang: } 10 \mathrm{~mm}+1 \mathrm{ppm} \text {, P.đứng: } \\
15 \mathrm{~mm}+1 \mathrm{ppm}\end{array}$ \\
\hline 7 & $\begin{array}{l}\text { Độ chính xác khi di } \\
\text { chuvển }\end{array}$ & $0,03 \mathrm{~m} / \mathrm{s}$ \\
\hline 8 & $\begin{array}{l}\text { Thời gian xác định tọa độ } \\
\text { lần đầu }\end{array}$ & Nhỏ hơn 50 giây \\
\hline 9 & Thời gian khởi động & Nhỏ hơn 10 giây \\
\hline 10 & Cổng giao tiếp & $\begin{array}{l}\text { Cồng COM DB9-RS232, } \\
\text { Bluetooth }\end{array}$ \\
\hline 11 & Nguồn điện & $5 \mathrm{~V}$ \\
\hline 12 & Công suất & $2.5 \mathrm{~W}$ \\
\hline
\end{tabular}


Bảng 2: Thông số thiết bị bay không người lái

\begin{tabular}{|c|c|c|}
\hline STT & Mô tả & Thông số \\
\hline 1 & Cất cánh và hạ cánh & VTOL \\
\hline 2 & Nguồn & PIN \\
\hline 3 & Tốc độ bay & $72 \mathrm{~km} / \mathrm{h}$ (TAS) \\
\hline 4 & Sức chống gió & $10 \mathrm{~m} / \mathrm{s}$ \\
\hline 5 & Tốc độ tối đa & $5 \mathrm{~m} / \mathrm{s}$ ( ơ độ cao 500 m) \\
\hline 6 & Bay xa tối thiểu & $3500 \mathrm{~m}$ \\
\hline 7 & Thời gian ca bay & $\begin{array}{l}90 \text { phút (Độ cao } 100 \mathrm{~m} \text { trên mặt } \\
\text { biển) }\end{array}$ \\
\hline 8 & Trọng lượng & $7 \mathrm{~kg}$ \\
\hline 9 & $\begin{array}{l}\text { Trọng lượng cất cánh tối } \\
\text { đa }\end{array}$ & $13 \mathrm{~kg}$ \\
\hline 10 & Tải trọng mang theo vật & $1.3 \mathrm{~kg}$ \\
\hline 11 & Mức ồn & 45dba@200mAGL \\
\hline 12 & Công suát đầu ra & $1 \mathrm{~W}$ \\
\hline 13 & Giải tần số & $902-928 \mathrm{Mhz}$ \\
\hline 14 & Mã hóa dữ liệu & 128bit AES encryption \\
\hline 15 & Spread Spectrum & FHSS \\
\hline 16 & Tốc độc truyền & 115200 \\
\hline 17 & Phạm vi truyền tài & $60 \mathrm{~km}$ \\
\hline 18 & Lỗi CRC & 32 bit $\mathrm{CRC}, \mathrm{ARQ}$ \\
\hline 19 & Camera Sony ILCE 5100 & $6000 \times 4000$ \\
\hline
\end{tabular}

Sau khi xây dựng hệ thống mốc khống chế, tiến hành bay chụp bãi hiệu chuẩn sử dụng hệ thống thiết bị GNSS-IMU-UAV. Trong quá trình xác định các thông số độ lệch chuẩn của hệ thống thiết bị, các mốc khống chế mặt bằng, độ cao trên bãi hiệu chuẩn được xác định tọa độ, độ cao từ mô hình và được coi như là các điểm kiểm tra để đánh giá kết quả.

Bảng 3: Các thông số tuyến bay hiệu chuẩn

\begin{tabular}{|c|l|l|}
\hline STT & Diện tích khu đo & $64 \mathrm{ha}$ \\
\hline 1 & Chiều dài & $14.58 \mathrm{~km}$ \\
\hline 2 & Khoảng cách giữa các ảnh & $83.2 \mathrm{~m}$ \\
\hline 3 & Độ phân giải mặt đất & $8.02 \mathrm{~cm}$ \\
\hline 4 & Độ cao bay & $300 \mathrm{~m}$ \\
\hline 5 & Camera & Sony ILCE 5100 \\
\hline
\end{tabular}

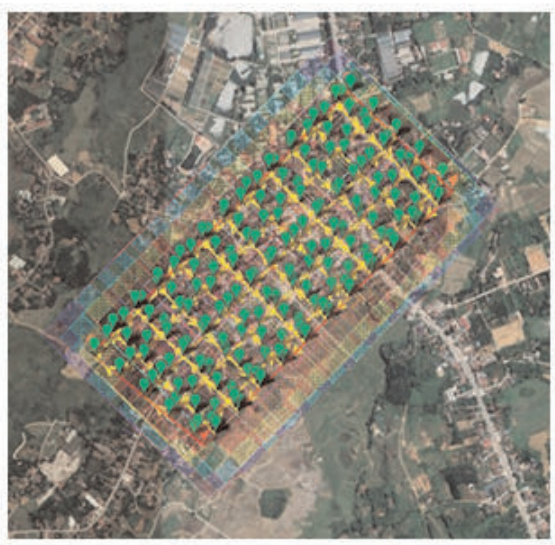

Hình 3: So đồ thiết kế tuyến bay hiệu chuẩn
2.3. Đánh giá thử nghiệm và lựa chọn phương án xử lý dĩ liệu phù hợp

Sau khi thực hoàn thành công tác bay chụp, các dữ liệu thu được bao gồm: dữ liệu ảnh UAV, dữ liệu GNSS-PPK, dữ liệu IMU. Quá trình xử lý dữ liệu được tiến hành theo 3 phương án:

- Phương án 1: Xác định các yếu tố định hướng ngoài của ảnh từ dữ liệu ảnh chụp UAV của máy ảnh, kết hợp với dữ liệu đo khống chế ảnh. Đây là phương pháp truyền thống, đang được sử dụng phổ biến.

- Phương án 2: Xác định các yếu tố định hướng ngoài của ảnh từ dữ liệu ảnh UAV của máy ảnh kết hợp với dữ liệu GNSS thu nhận dữ liệu theo công nghệ trạm tham chiếu ảo (VRS), IMU gắn trên UAV. Đây cũng là phương án tích hợp của đề tài.

- Phương án 3: Xác định các yếu tố định hướng ngoài của ảnh từ dữ liệu ảnh UAV của máy ảnh kết hợp với dữ liệu GNSS, IMU gắn trên UAV và 4 điểm khống chế ảnh.

\section{a) Kết quả xử lý theo phrơng án 1 :}

Kết quả xử lý theo phương án 1 cho thấy, tọa độ tâm ảnh UAV chưa qua xử lý chênh lệch so với tọa độ sau xử lý trung bình khoảng 22.5 mét. 
Bảng 4: So sánh tọa độ điểm kiểm tra sau khi xủ lý theo phwong án 3 với giá trị đo đạc ngoài thục địa

\begin{tabular}{|c|c|c|c|c|c|c|c|c|c|}
\hline \multirow[b]{2}{*}{ Tên điềm } & \multicolumn{3}{|c|}{$\begin{array}{l}\text { Tọa độ điềm kiềm tra sau khi xừ ly theo } \\
\text { Phurong an } 3\end{array}$} & \multicolumn{3}{|c|}{$\begin{array}{c}\text { Tọa độ điểm kiểm tra đo đạc } \\
\text { ngoài thục địa }\end{array}$} & \multicolumn{3}{|c|}{ Sai số vị trí } \\
\hline & $y(m)$ & $\mathrm{x}(\mathrm{m})$ & $h(m)$ & $y(m)$ & $x(m)$ & $h(m)$ & $\begin{array}{c}\text { deltay } \\
(\mathrm{m})\end{array}$ & $\begin{array}{c}\operatorname{celtax}^{\text {delm }} \\
(\mathrm{m})\end{array}$ & $\begin{array}{l}\text { delta h } \\
(\mathrm{m})\end{array}$ \\
\hline 101 & 555178.624 & 2317924.030 & 24.826 & 555178.673 & 2317924.043 & 24.537 & 0.049 & 0.013 & -0.285 \\
\hline 102 & 555637.520 & 2318487.007 & 22.424 & 555637.590 & 2318487.043 & 22.412 & 0.070 & 0.036 & -0.012 \\
\hline 103 & 555639.216 & 2318475.311 & 22.108 & 555639.156 & 2318475.256 & 22.115 & -0.060 & -0.055 & 0.007 \\
\hline 104 & 555406.517 & 2318485.596 & 27.241 & 555406.485 & 2318485.623 & 27.506 & -0.032 & 0.027 & $0.26=$ \\
\hline 105 & 555057.037 & 2317994.995 & 31.149 & 555056.994 & 2317994.946 & 31.168 & -0.043 & -0.049 & 0.019 \\
\hline 106 & 554993.813 & 2317745.028 & 31.713 & 554993.898 & 2317744.974 & 31.794 & 0.085 & -0.054 & 0.081 \\
\hline $\mathrm{KCA} 01$ & 555323.080 & 2318333.360 & 28.844 & 555323.097 & 2318333.340 & 28.848 & 0.017 & -0.020 & 0.004 \\
\hline $\mathrm{KCA} 02$ & 555322.416 & 2318334.090 & 28.854 & 555322.415 & 2318334.080 & 28.807 & -0.001 & -0.010 & -0.04 \\
\hline KCA 03 & 555360.055 & 2318373.719 & 28.111 & 555360.014 & 2318373.720 & 28.069 & -0.041 & 0.001 & -0.042 \\
\hline KCA 04 & 555353.588 & 2318372.906 & 28.087 & 555353.629 & 2318372.909 & 28.103 & 0.041 & 0.003 & 0.016 \\
\hline KCA 05 & 555416.764 & 2318320.559 & 28.476 & 555416.785 & 2318320.508 & 28.508 & 0.021 & -0.051 & 0.032 \\
\hline $\mathrm{KCA} 08$ & 555480.006 & 2318297.884 & 26.434 & 555480.020 & 2318297.862 & 26.403 & 0.014 & -0.022 & -0.031 \\
\hline $\mathrm{KCA} 09$ & 555477.086 & 2318291.992 & 26.522 & 555477.103 & 2318291.997 & 26.473 & 0.017 & 0.005 & -0.049 \\
\hline KCA 11 & 555346.356 & 2318172.985 & 30.168 & 555346.387 & 2318172.971 & 30.171 & 0.031 & -0.014 & 0.003 \\
\hline $\mathrm{KCA} 12$ & 555344.493 & 2318169.701 & 30.180 & 555344.521 & 2318169.698 & 30.185 & 0.028 & -0.003 & 0.005 \\
\hline KCA 16 & 555509.452 & 2318228.587 & 23.737 & 555509.495 & 2318228.573 & 23.696 & 0.043 & -0.014 & -0.041 \\
\hline KCA 17 & 555513.662 & 2318225.532 & 23.695 & 555513.688 & 2318225.571 & 23.702 & 0.026 & 0.039 & 0.007 \\
\hline KCA 18 & 555517.032 & 2318266.397 & 23.563 & 555517.045 & 2318266.424 & 23.544 & 0.013 & 0.027 & -0.019 \\
\hline KCA 19 & 555514.623 & 2318268.221 & 23.558 & 555514.664 & 2318268.197 & 23.590 & 0.041 & -0.024 & 0.032 \\
\hline KCA 20 & 555531.930 & 2318297.099 & 23.517 & 555531.902 & 2318297.073 & 23.496 & -0.028 & -0.026 & -0.021 \\
\hline $\mathrm{KCA} 21$ & 555547.373 & 2318313.069 & 23.401 & 555547.329 & 2318313.052 & 23.342 & -0.044 & -0.017 & -0.059 \\
\hline KCA 22 & 555546.565 & 2318285.456 & 21.435 & 555546.537 & 2318285.465 & 21.468 & -0.028 & 0.009 & 0.033 \\
\hline $\mathrm{KCA} 23$ & 555544.990 & 2318286.631 & 21.507 & 555544.978 & 2318286.590 & 21.454 & -0.012 & -0.041 & -0.053 \\
\hline KCA 24 & 555545.076 & 2318236.739 & 20.397 & 555545.063 & 2318236.760 & 20.376 & -0.013 & 0.021 & -0.021 \\
\hline KCA 25 & 555502.565 & 2318257.593 & 23.545 & 555502.577 & 2318257.639 & 23.576 & 0.012 & 0.046 & 0.031 \\
\hline KCA 26 & 555504.844 & 2318270.636 & 23.638 & 555504.848 & 2318270.609 & 23.594 & 0.004 & -0.027 & -0.044 \\
\hline KCA 13 & 555383.265 & 2318158.232 & 29.459 & 555383.317 & 2318158.206 & 29.411 & 0.052 & -0.026 & -0.048 \\
\hline KCA 31 & 555317.968 & 2318424.917 & 27.797 & 555318.000 & 2318424.961 & 27.871 & 0.032 & 0.044 & 0.074 \\
\hline KCA 28 & 555396.177 & 2318152.557 & 29.182 & 555396.180 & 2318152.482 & 29.135 & 0.003 & -0.075 & -0.047 \\
\hline 107 & 555398.542 & 2318141.655 & 29.027 & 555398.501 & 2318141.652 & 29.011 & -0.041 & -0.003 & -0.016 \\
\hline 108 & 555396.525 & 2318138.372 & 29.070 & 555396.550 & 2318138.409 & 29.048 & 0.025 & 0.037 & -0.022 \\
\hline 109 & 555420.024 & 2318190.368 & 28.989 & 555420.033 & 2318190.327 & 29.001 & 0.009 & -0.041 & 0.012 \\
\hline KCA 35 & 555356.379 & 2318295.149 & 29.444 & 555356.381 & 2318295.140 & 29.470 & 0.002 & -0.009 & 0.026 \\
\hline $\mathrm{KCA} 36$ & 555357.847 & 2318293.746 & 29.443 & 555357.812 & 2318293.767 & 29.452 & -0.035 & 0.021 & 0.005 \\
\hline $\mathrm{KCA} 37$ & 555357.430 & 2318302.926 & 29.193 & 555357.371 & 2318302.956 & 29.227 & -0.059 & 0.030 & 0.034 \\
\hline KCA 38 & 555344.358 & 2318290.872 & 29.659 & 555344.372 & 2318290.882 & 29.687 & 0.014 & 0.010 & 0.028 \\
\hline $\mathrm{KCA} 39$ & 555270.071 & 2318264.481 & 30.574 & 555270.038 & 2318264.518 & 30.538 & -0.033 & 0.037 & -0.036 \\
\hline
\end{tabular}

Trong đó, sai số mặt bằng đạt 3.7 mét và độ cao đạt $22.317 \mathrm{~m}$. Ngoài ra, trong 6 yếu tố định hướng ngoài của ảnh bao gồm tọa độ tâm ảnh và 3 góc xoay thì dữ liệu ảnh của UAV đơn thuần chỉ có tọa độ tâm ảnh và không có 3 góc xoay cho nên các góc xoay sau xử lý được xác định phương pháp nắn ảnh lập thể dựa vào các điểm khống chế ảnh. Do vậy việc bố trí các điểm khống chế ảnh mặt đất yêu cầu phải phủ trùm và rải đều khu đo với mật độ tương đối dày. Hơn nữa, đối với những khu vực độ che phủ cao, hoặc không xác định được điểm khống chế trên ảnh 
thì độ chính xác sẽ bị giảm.

b) Kết quả xư lý theo phương án 2:

So sánh giá trị các yếu tố định hướng ngoài của ảnh khi xác định bằng dữ liệu GNSS-IMU và khi xác định bằng toàn bộ khống chế ảnh rải đều khu đo cho kết quả sai mặt bằng đạt 0.72 mét, sai số độ cao đạt 2.073 mét, sai số góc xoay pitch, roll, yaw rất nhỏ $\left(<1^{0}\right)$.

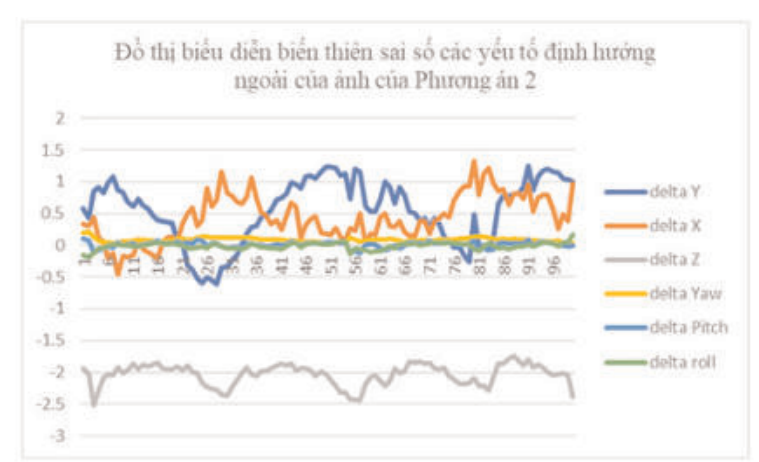

c) Kết quả xủ lý theo phioong án 3:

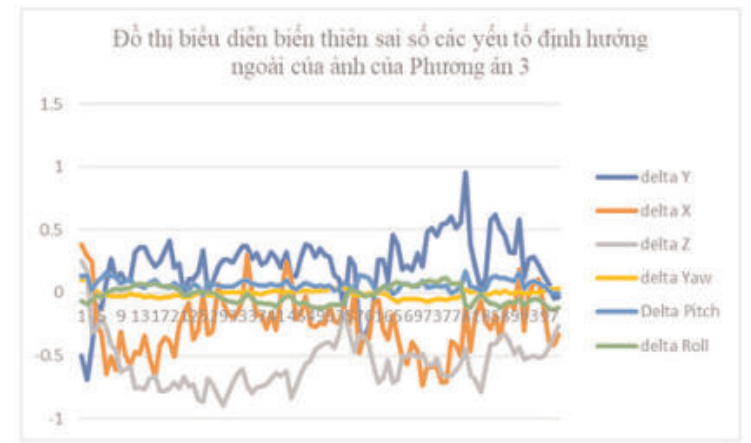

Nhận xét: Kết quả xử lý theo phương án 3 cho kết quả tọa độ tâm ảnh gần đúng nhất với sai số mặt bằng đạt 10-20 cm, sai số độ cao đạt 50-
$70 \mathrm{~cm}$. Việc sử dụng dữ liệu GNSS-IMU kết hợp với dữ liệu ảnh UAV đã xác định được gần đúng tọa độ tâm ảnh chính xác do vậy việc đo khống chế ảnh có thể giảm lên đến $80 \%$. (Xem bảng 4)

\section{Kết luận}

Qua quá trình thực nghiệm, tác giả nhận thấy rằng việc sử dụng dữ liệu GNSS kết hợp IMU để xử lý tọa độ tâm ảnh có thể giảm tới $80 \%$ số điểm khống chế ảnh cần đo đạc so với trước đây. Công nghệ này đã góp phần nâng cao mức độ tự động hóa trong công tác sử dụng thiết bị bay không người lái (UAV) trong đo đạc thành lập bản đồ địa hình. $O$

\section{Tài liệu tham khảo}

[1]. Dieu Tien Bui, Van Cam Nguyen, Nguyen Quang Minh, 2016, Xây dựng mô hình số bề mặt và bản đồ trực ảnh sử dụng công nghệ đo ảnh máy bay không người lái (UAV), Hội nghị khoa học đo đạc bản đồ và ứng phó với biến đổi khí hậu tháng 7/2016, trang 18-25.

[2]. Trần Trung Anh, 2018, Kết hợp công nghệ UAV, RTK và SES trong thành lập bản đồ địa hình tỷ lệ lớn vùng rừng ngập mặn ven biển, Hội nghị toàn quốc khoa học trái đất và tài nguyên với phát triển bền vững 2018, trang 3441.

[3]. Phan Bảo Châu, Ngô Khánh Hiếu, Nguyễn Vĩnh Bảo, 2014, Phát triển hệ thống dẫn đường tích hợp GPS/INS cho máy bay mô hình, Tạp chí KH\&CN Tập 17, Số K7-2014, trang 2834.0

\section{Summary}

Integrating IMU and GNSS devices to receive data using virtual reference station technology (VRS) on unmanned aerial vehicles (UAVs) for topographic mapping.

\section{Luu Hai Au, Dang Xuan Thuy, Ngo Thi Lien}

The topographic mapping application of unmanned aerial vehicles (UAV) has been applied and is gradually becoming more popular. Determining the exact factors external orientation of photos is one of the factors determining the accuracy of topographic maps should be established instead of setting the image control network. This article discusses the issue of integrating GNSS-IMU devices on unmanned aerial vehicles (UAVs) in combination with virtual reference station technology (VRS) to identify outward-facing elements of an image with accurately ensure the creation of a large scale terrain map with the maximum number of image control points. $\bigcirc$ 